\title{
Conducta prosocial y motivación académica en estudiantes españoles de Educación Secundaria Obligatoria*
}

Prosocial behavior and academic motivation in Spanish High School Students

Recibido: febrero 2 de 2010 | Revisado: abril 15 de 2010 | Aceptado: mayo 16 de 2010

\author{
CÁNDIDO J. INGLÉS *** \\ Agustín E. MartíneZ-GONZÁLEZ \\ Universidad Miguel Hernández de Elche, España \\ ANTONIO VALLE ${ }^{* * *}$ \\ Universidad de A Coruña, España \\ José M. GARCÍA-FERNÁNDEZ ${ }^{* * * * *}$ \\ Universidad de Alicante, España \\ Cecilia Ruiz-Esteban ${ }^{* * * * *}$ \\ Universidad de Murcia, España
}

SICI: 1657-9267(201108) 10:2<451:CPMAEE >2.0.TX;2-V

Para citar este artículo. Inglés, C. J., MartínezGonzález, A. E., Valle, A., García-Fernández, J. M. \& Ruiz-Esteban, C. (2011). Conducta prosocial y motivación académica en estudiantes españoles de Educación Secundaria Obligatoria. Universitas Psychologica, 10 (2), 451-465.

Artículo de investigación

** Dpto. de Psicología de la Salud, Avda. de la Universidad s/n, Elche.03202, España.E-mails: cjingles@ umh.es; cpcmayor@yahoo.es

Research-ID: Martínez-González, A. E., E-1409. 2011

**** Departamento de Psicoloxía Evolutiva e da Educación, Campus de Elviña s/n 15.071. A Coruña, España.E-mail: vallar@udc.es

***** Dpto. Psicología Evolutiva y Didáctica, Apdo. Correos: 99. 03080, Alicante, España. E-mail: josemagf@ua.es

******* Dpto. de Psicología Evolutiva y de la Educación. Campus de Espinardo. 30100, Murcia, España E-mail: cruiz@um.es

\section{RESUMEN}

Este estudio analizó la relación entre conducta prosocial y metas académicas en una muestra de 2.022 estudiantes españoles. La conducta prosocial fue medida con la escala de conducta prosocial del Teenage Inventory of Social Skills (TISS) y las metas académicas mediante el Achievement Goal Tendencies Questionnaire (AGTQ). Los resultados revelaron que los estudiantes con alta conducta prosocial presentaron puntuaciones significativamente más altas en metas de aprendizaje y logro. La conducta prosocial fue un predictor positivo y estadísticamente significativo de metas de aprendizaje y logro. Además, las metas de aprendizaje y logro fueron predictores positivos y estadísticamente significativos de la conducta prosocial, mientras que las metas de refuerzo social fueron un predictor negativo y estadísticamente significativo de la conducta prosocial.

Palabras clave autores

Adolescencia, conducta prosocial, educación secundaria, metas académicas.

Palabras clave descriptores

Investigación psicológica, test psicológico, comportamiento social, comportamiento de grupo, relaciones interpersonales, afectividad, voluntad de realización.

\section{A B S T R A C T}

This study analyzed the relationship between prosocial behaviour and academic goals in a sample of 2,022 Spanish compulsory secondary education students. The prosocial behaviour was measured with the Prosocial Behaviour scale of the Teenage Inventory of Social Skills (TISS) and academic goals were measured with the Achievement Goal Tendencies Questionnaire (AGTQ). The results revealed that students with high prosocial behaviour presented higher significantly scores in learning and performance goals. The prosocial behaviour was a positive and statistically significant predictor of learning and performance goals. Furthermore, learning and performance goals were positive and statistically significant predictors of the prosocial behaviour, whereas social reinforcement goals were a negative and statistically significant predictor of prosocial behaviour.

Key words authors

Adolescence, prosocial behavior, secondary education, academic goals.

Key words plus

Psychological Research, Psychological Tests, Social Behaviour, Group Behaviour, Interpersonal Relations, Emotions, Achievement Motivation. 
La evidencia empírica previa relativa a las relaciones entre conducta prosocial autopercibida y metas académicas en estudiantes de Educación Secundaria es prácticamente inexistente, aun cuando actualmente ambas variables son consideradas factores clave para el ajuste personal, social y académico de los estudiantes (Meece, Anderman \& Anderman, 2006; Wentzel, 2005; Wentzel \& Watkins, 2002). Por ello, el presente estudio pretende contribuir al conocimiento sobre la relación entre ambos constructos, analizando las diferencias en metas académicas entre estudiantes identificados como prosociales y no prosociales, y examinando la capacidad predictiva recíproca entre conducta prosocial y metas académicas en una muestra de estudiantes españoles de Educación Secundaria Obligatoria (ESO). A continuación, se presentan algunas de las razones que han motivado la realización de este estudio.

\section{Importancia de la conducta prosocial en ESO}

La conducta prosocial es definida como una conducta voluntaria dirigida a establecer relaciones positivas, empáticas, cooperativas y responsables, con el fin de beneficiar a otros (Eisenberg, Fabes $\&$ Spinrad, 2006; Wentzel, 2005).

Tradicionalmente, lo que más ha preocupado a los investigadores en Educación y Psicología ha sido el estudio de la conducta agresiva y antisocial en la adolescencia (Inglés et al., 2008). Sin embargo, esta tendencia está cambiando en los últimos años y numerosos autores han comenzado a estudiar la conducta prosocial, ya que esta desempeña un papel fundamental en la formación de relaciones interpersonales positivas y en la aceptación por los iguales (Eisenberg et al., 2006; Gilman \& Anderman, 2006; Inglés, Delgado, García-Fernández, Ruiz-Esteban \& Díaz-Herrero, 2010), así como en el ajuste escolar y el éxito académico (Gilman \& Anderman, 2006; Inglés, Benavides et al., 2009; Wentzel, 2005). Además, la conducta prosocial puede ejercer un efecto inhibitorio sobre las conductas sociales negativas, tales como, agresividad o retraimiento social, convirtiéndose en un factor clave para la promoción de la competencia social y académica en los centros educativos (Eisenberg et al., 2006; Wentzel, 2005). En esta línea, Inglés et al. (2008) indicaron que los responsables de políticas educativas y, en última instancia, las escuelas y los educadores, deberían considerar la elevada prevalencia de jóvenes prosociales españoles como un recurso preventivo y terapéutico con el fin de promover el aprendizaje y/o mejora de las habilidades sociales, así como tratar a los estudiantes con problemas de agresividad y ansiedad social. Además, la investigación ha demostrado que el modelado de conductas sociales realizado por iguales socialmente hábiles y prosociales, es una de las técnicas de intervención cognitivo-conductuales que mejoran sustancialmente la eficacia del entrenamiento en habilidades sociales aplicado a adolescentes o estudiantes de Educación Secundaria (Rosa et al., 2002).

\section{Importancia de la motivación académica en ESO}

El incremento significativo en las tasas de fracaso escolar y abandono prematuro de los estudios por parte de los estudiantes españoles de ESO, ha dado lugar a que ambas cuestiones se conviertan en problemas importantes a los que debe enfrentarse el sistema educativo español (Ministerio de Educación y Ciencia, 2007). Este fenómeno preocupante ha conducido a profesores, psicólogos educativos, pedagogos, psicopedagogos, responsables de la política educativa e investigadores a reconsiderar qué factores están implicados en la falta de interés de los estudiantes por las tareas escolares (Pintrich, 2003). En esta línea, diversas investigaciones han indicado que la motivación académica es una factor relevante en la predicción del rendimiento académico (Inglés, García-Fernández et al., 2009; Steinmayr \& Spinath, 2009) y en la prevención del fracaso escolar y el abandono prematuro de los estudios (Alderman, 2008; Meece et al., 2006; Valle et al., 2010), ya que actúa facilitando la aparición de estrategias de aprendizaje que mejoran el rendimiento académico de los alumnos a través de un análisis más profundo de la información (Covington, 2000). 


\section{Relaciones entre conducta prosocial y motivación académica}

La evidencia empírica previa ha puesto de manifiesto la existencia de una relación significativa entre conducta prosocial y diversas variables psicoeducativas, tales como rendimiento escolar (Inglés, Benavides et al., 2009; Wentzel, 2003) y autoeficacia académica (Bandura, Caprara, Barbaranelli, Gerbino \& Pastorelli, 2003). Sin embargo, en la actualidad, existen pocos estudios internacionales en los que se analiza la relación entre comportamiento prosocial y motivación académica. Así, Wentzel y Asher (1995), usando una muestra de escolares de Educación Primaria, encontraron que las percepciones de los iguales sobre las conductas prosociales de sus compañeros de clase se relacionaron positivamente con niveles elevados de motivación académica. Posteriormente, Gilman y Anderman (2006) encontraron resultados similares en una muestra de estudiantes de Educación Secundaria. Concretamente, estos autores hallaron que la conducta prosocial de los adolescentes valorada por los iguales se relacionó positiva y significativamente con niveles altos de motivación intrínseca. Por el contrario, los adolescentes con baja motivación intrínseca fueron valorados por sus iguales como más proclives a comenzar peleas y menos interesados por ayudar a otros y a realizar correctamente sus tareas escolares. Así, consistente con los hallazgos de Wentzel y Asher (1995), Gilman y Anderman (2006) encontraron que las relaciones con los iguales parecen tener un papel significativo, cuando se pretende comprender la motivación entre estudiantes de Educación Secundaria.

\section{El presente estudio}

Aunque la investigación previa sugiere, tal y como ha sido indicado, que las relaciones entre funcionamiento social y motivación académica están intrínsecamente entrelazadas (Gilman \& Anderman, 2006; Wentzel, 2005; Wentzel \& Watkins, 2002), en la actualidad, tan sólo existen dos estudios internacionales en los que se ha analizado la relación entre comportamiento prosocial y motivación académica (Gilman \& Anderman, 2006; Wentzel \& Asher, 1995), no habiéndose realizado, hasta ahora, ningún estudio en España respecto a esta cuestión, algo sorprendente si se considera que la mayoría de teorías actuales explican la motivación como un conjunto de conductas dirigidas hacia una meta, las cuales son moduladas por procesos cognitivos y sociales (Furrer \& Skinner, 2003; Wentzel, 2005). Además, en los dos estudios previos tan sólo se evaluó, mediante nominaciones por los iguales, la motivación intrínseca o metas de aprendizaje, es decir, la tendencia de los estudiantes por aprender con el propósito de adquirir conocimientos y aumentar su competencia, no incluyendo medidas de motivación extrínseca tales como las metas de logro (o tendencia de los estudiantes por aprender, con la intención de obtener buenas notas en los exámenes y avanzar en los estudios) y las metas de refuerzo social (o tendencia de los estudiantes por aprender para obtener juicios de aprobación y evitar el rechazo de padres y profesores).

Por tanto, este estudio trata de contribuir al conocimiento sobre las relaciones entre conducta prosocial y metas académicas en estudiantes españoles de ESO, mediante la consecución de dos objetivos. El primer objetivo consistió en analizar las diferencias en motivación académica (metas de aprendizaje, metas de logro y metas de refuerzo social) entre estudiantes identificados como prosociales y no prosociales. El segundo objetivo consistió en examinar la capacidad predictiva recíproca entre la conducta prosocial y los tres tipos de metas académicas propuestas por Hayamizu y Weiner (1991).

En diferentes estudios se ha encontrado que: (a) la conducta prosocial se relaciona positiva y significativamente con la motivación intrínseca o metas de aprendizaje (Gilman \& Anderman, 2006; Wentzel \& Asher, 1995); (b) la conducta prosocial se relaciona positiva y significativamente con las metas de logro o rendimiento, en tanto que actúa como una variable mediadora entre la competencia social con los iguales y los logros académicos, contribuyendo a la mejora de ambos dominios (Wentzel, 2005) y como un predictor positivo y 
estadísticamente significativo del éxito académico (Inglés, Benavides et al., 2009). Además, (c) el éxito académico es, a su vez, un predictor positivo y estadísticamente significativo de la conducta prosocial (Inglés, Benavides et al., 2009); (d) los estudiantes con éxito académico presentan tanto metas académicas (metas de aprendizaje y logro) como sociales (comportamiento prosocial y socialmente responsable), es decir, metas múltiples (Wentzel, 2005); (e) la búsqueda de metas prosociales y socialmente responsables ha sido relacionada con la búsqueda de metas de aprendizaje y con el logro de buenas calificaciones académicas (metas de logro), y viceversa (Wentzel, 2005) y (f) el éxito académico es más probable cuando aumentan las puntuaciones en metas de aprendizaje y metas de logro (Inglés, García-Fernández et al., 2009). Con base en estos resultados se espera que tanto en la muestra total como en ambos géneros y en todos los cursos académicos:

1) Los estudiantes con alta conducta prosocial, en comparación con sus iguales con baja prosociabilidad, presenten niveles significativamente más altos en metas de aprendizaje y metas de logro.

2) La conducta prosocial sea un predictor positivo y estadísticamente significativo de puntuaciones altas en metas de aprendizaje y de logro.

3) Las puntuaciones en metas de aprendizaje y de logro sean predictores positivos y estadísticamente significativos de la probabilidad de alta conducta prosocial.

En cuanto a la relación entre conducta prosocial y metas de refuerzo social, es razonable esperar que:

4) Los estudiantes con alta conducta prosocial, en comparación con sus iguales con baja conducta prosocial, presenten niveles significativamente más bajos de metas de refuerzo social, tanto en la muestra total como en ambos géneros y en todos los cursos académicos.

5) La conducta prosocial sea un predictor negativo y estadísticamente significativo de una puntuación alta en metas de refuerzo social, tanto en la muestra total como en ambos géneros y en todos los cursos académicos.

6) La puntuación en metas de refuerzo social sean un predictor negativo y estadísticamente significativo de la probabilidad de presentar alta conducta prosocial, tanto en la muestra total como en ambos géneros y en todos los cursos académicos.

La justificación teórica y empírica relativa a la formulación de estas tres últimas hipótesis es aún más indirecta que en el caso de las metas de aprendizaje y logro. En cualquier caso, estas han sido elaboradas teniendo en cuenta las siguientes consideraciones: (a) diversos autores han encontrado que el éxito académico es menos probable cuando aumentan las puntuaciones en metas de refuerzo social (Inglés, García-Fernández et al., 2009) y; (b) los estudiantes prosociales se caracterizan por ser empáticos, cooperativos (no competitivos), socialmente competentes y responsables hacia los demás, así como por tener más amigos y ser aceptados por y populares entre los iguales y profesores (Inglés et al., 2010; Wentzel, 2005). Por ello, es previsible suponer que los estudiantes prosociales, comparados con sus iguales no prosociales, adopten en menor medida metas de refuerzo social, tales como "estudio porque quiero que la gente me vea lo listo/a que soy" o "estudio porque me gusta sacar mejores notas que mis compañeros".

\section{Método}

\section{Participantes}

Se realizó un muestreo aleatorio por conglomerados (zonas geográficas de la región de Murcia y la provincia de Alicante: centro, norte, sur, este y oeste). Con el fin de que todas las zonas geográficas estuvieran representadas se seleccionaron aleatoriamente 20 centros de áreas rurales y urbanas, 14 públicos y 6 privados. Cada zona geográfica estuvo representada por una media de dos centros. Una vez determinados los centros del estudio, se seleccionaron aleatoriamente cuatro aulas, computándose aproximadamente 120 sujetos por centro. 
El total de sujetos reclutados fue 2.267 estudiantes de $1^{\circ}$ a $4^{\circ}$ de $\mathrm{ESO}$, de los que 116 (5,12 $\%)$ fueron excluidos por errores u omisiones en sus respuestas, por no obtener por escrito el consentimiento informado de los padres para participar en la investigación y 129 (5,69 \%) fueron excluidos por ser extranjeros con importantes déficits en el dominio de la lengua española. Por tanto, la muestra definitiva se compuso de 2.022 estudiantes, con un rango de edad de 12 a 16 años $(M=13,81 ; D T$ $=1,35)$. La distribución de la muestra por género y curso académico fue la siguiente: 1.033 varones, 989 mujeres, 576 ( $1^{\circ}$ de ESO), 505 ( $2^{\circ}$ de ESO), 502 (3을 ESO) y 439 (4을 ESO). El 86,30 \% de los estudiantes fueron no repetidores. La composición étnica de la muestra fue la siguiente: 88,9 $\%$ españoles, 6,34 \% hispanoamericanos, 3,37 \% resto de Europa, 0,75 \% asiáticos y 0,64 \% árabes.

\section{Instrumentos}

Inventario de Habilidades Sociales para Adolescentes (Teenage Inventory of Social Skills [TISS] de Inderbitzen \& Foster, 1992)

El TISS evalúa la conducta prosocial y agresiva de los adolescentes en las relaciones con sus iguales. Consta de 40 ítems agrupados en dos escalas: Conducta Prosocial y Conducta Antisocial. Los ítems se valoran mediante una escala Likert de 6 puntos $(1=$ no me describe nada; 6 = me describe totalmente). El TISS ofrece dos puntuaciones, una para conducta prosocial y otra para conducta antisocial, las cuales se obtienen sumando los valores asignados por los sujetos a los 20 ítems que componen cada dimensión. Puntuaciones altas indican elevada conducta prosocial y agresiva.

El TISS es uno de los instrumentos de evaluación de las habilidades sociales con mejores garantías psicométricas en población anglo e hispanoparlante. La estructura bi-factorial de la versión española del TISS fue confirmada por Inglés, Hidalgo, Méndez e Inderbitzen (2003) usando una muestra de adolescentes españoles. Además, Inglés et al. (2003) encontraron que los coeficientes de fiabilidad (consistencia interna) fueron satisfactorios y semejantes a los hallados por los autores originales en población adolescente norteamericana (Inderbitzen \& Foster, 1992). En este estudio se utilizó únicamente la escala de Conducta Prosocial, siendo su consistencia interna (alfa de Cronbach) 0,90.

Cuestionario de Evaluación de Metas Académicas (CEMA) (Achievement Goal Tendencies Questionnaire [AGTQ] de Hayamizu \& Weiner, 1991)

El Cuestionario de Evaluación de Metas Académicas (CEMA) es la adaptación española del AGTQ, y se compone de 20 ítems, agrupados en tres factores identificados mediante análisis factorial exploratorio por Hayamizu y Weiner (1991): Metas de Aprendizaje (MA), Metas de Refuerzo Social (MRS) y Metas de Logro (ML). La fiabilidad (consistencia interna) y el análisis de relaciones con otras variables psicoeducativas apoyó la validez de constructo del CEMA.

Las propiedades psicométricas de la versión española del CEMA han sido examinadas por varios investigadores usando distintas muestras de estudiantes de Educación Secundaria y Educación Superior (véanse Inglés, García-Fernández et al., 2009, para una revisión). Estos estudios apoyaron la estructura trifactorial del CEMA, obteniendo, además, otros indicadores adecuados de fiabilidad y validez de constructo del cuestionario. En este estudio, los coeficientes alfa de Cronbach para las escalas del CEMA fueron: 0,80 (MA), 0,77 (MRS) y 0,74 (ML).

\section{Procedimiento}

Se llevó a cabo una entrevista con los directores y psicopedagogos de los centros participantes para exponer los objetivos de la investigación, describir el instrumento de evaluación, pedir los permisos pertinentes y promover su colaboración. Posteriormente, se celebró una reunión con los padres para explicarles el estudio y solicitar el consentimiento informado por escrito autorizando a sus hijos a participar en la investigación. Los cuestionarios fueron contestados de forma colectiva, voluntaria y anónima en el aula, asignando previamente un número de identificación a las hojas de respuesta, 
las cuales fueron posteriormente corregidas mediante ordenador. Los investigadores estuvieron presentes durante la administración de la prueba para proporcionar ayuda si era necesaria y verificar la cumplimentación independiente por parte de los participantes.

\section{Análisis estadísticos}

La identificación de estudiantes con baja y alta conducta prosocial se estableció, una vez comprobado que la distribución de las puntuaciones de los participantes del estudio se ajustaba a la distribución teórica esperada en la escala de Conducta Prosocial del TISS. Así, la muestra general $(\mathrm{N}=2.022)$ se dividió en dos grupos: (a) sujetos con alta conducta prosocial: puntuaciones iguales o superiores al centil $75\left(n_{1}=540 ; 26,71 \%\right)$ y; (b) sujetos con baja conducta prosocial: puntuaciones iguales o inferiores al centil $25\left(n_{2}=518 ; 25,62 \%\right)$.

Para la consecución de los objetivos planteados en el presente estudio, los análisis estadísticos fueron realizados tanto para la muestra total como para los grupos de género y cursos académicos, ya que investigaciones previas han revelado diferencias de género y curso estadísticamente significativas en conducta prosocial (Inglés et al., 2008) y en metas académicas (Delgado, Inglés, García-Fernández, Castejón \& Valle, 2010).

Para analizar las diferencias en metas de aprendizaje, metas de logro y metas de refuerzo social entre estudiantes con baja y alta conducta prosocial, se aplicó la prueba de significación estadística $t$ de Student. Debido al elevado tamaño muestral del estudio, el estadístico $t$ de Student puede detectar erróneamente diferencias estadísticamente significativas. Por esta razón se incluye, además, el índiced (diferencia media tipificada) propuesto por Hedges (1981), que permite valorar la magnitud o el tamaño del efecto de las diferencias encontradas. Su interpretación es sencilla: $0,20 \leq d \leq 0,50$ supone un tamaño del efecto bajo, mientras que $0,51 \leq d \leq 0,79$ es moderado y $d \geq 0,80$ es alto.

Para examinar la capacidad predictiva recíproca entre conducta prosocial y metas académicas, se realizaron análisis de regresión logística binaria siguiendo el procedimiento de regresión por pasos hacia delante basado en el estadístico de Wald. Por tanto, para determinar la capacidad predictiva de la conducta prosocial (predictor) sobre las metas académicas (criterio), las variables criterio fueron dicotomizadas. Así, una vez comprobado que las distribuciones de las puntuaciones de los sujetos del estudio se ajustaban a la distribuciones teóricas esperadas en las escalas de Metas de Aprendizaje, Metas de Refuerzo Social y Metas de Logro del CEMA, la muestra se dividió en seis grupos: (a) estudiantes con altas y bajas metas de aprendizaje: puntuaciones iguales o superiores al centil $75 \mathrm{e}$ iguales o inferiores al centil 25, respectivamente; (b) estudiantes con altas y bajas metas de refuerzo social: puntuaciones iguales o superiores al centil 75 e iguales o inferiores al centil 25 , respectivamente, y; (c) estudiantes con altas y bajas metas de logro: puntuaciones iguales o superiores al centil 75 e iguales o inferiores al centil 25, respectivamente. Además, para analizar la capacidad predictiva de las metas académicas (predictores) sobre la probabilidad de presentar alta conducta prosocial (criterio), las puntuaciones en conducta prosocial correspondientes a la escala de Conducta Prosocial del TISS fueron nuevamente dicotomizadas en: (a) alta conducta prosocial: puntuaciones iguales o superiores al centil 75 y; (b) baja conducta prosocial: puntuaciones iguales o inferiores al centil 25 .

El modelado logístico permite estimar la probabilidad de que ocurra un evento, suceso o resultado (por ejemplo, alta conducta prosocial) en presencia de uno o más predictores (por ejemplo, metas de aprendizaje, metas de refuerzo social y metas de logro). Esta probabilidad es estimada mediante el estadístico denominado odd ratio (OR). Si la OR es mayor que uno, entonces el evento es más probable que ocurra cada vez que el factor o variable independiente incrementa un punto. Si la $\mathrm{OR}$ es menor que uno, entonces el evento es menos probable que ocurra cada vez que el factor $o$ variable independiente incrementa un punto (De Maris, 2003). 


\section{Resultados}

Diferencias en metas académicas entre estudiantes con baja y alta conducta prosocial

La Tabla 1 muestra las puntuaciones medias y desviaciones típicas de los estudiantes con baja y alta conducta prosocial para la muestra total, el género y los cursos académicos en las tres escalas del CEMA. Respecto a la muestra total, los resultados indican que los alumnos con alta conducta prosocial puntúan significativamente más alto en Metas de Aprendizaje y en Metas de Logro que a los alumnos con baja conducta prosocial, siendo la magnitud de estas diferencias moderada en el pri-

TABLA 1

Metas académicas en estudiantes con baja y alta conducta prosocial

\begin{tabular}{|c|c|c|c|c|c|c|c|}
\hline \multirow{2}{*}{$\begin{array}{c}\text { Metas académicas (rango) } \\
\text { Muestra total }\end{array}$} & \multicolumn{2}{|c|}{ Baja conducta rosocial } & \multicolumn{2}{|c|}{ Alta conducta prosocial } & \multicolumn{3}{|c|}{ Significación estadística } \\
\hline & M & DT & M & DT & $t_{1056}$ & $p$ & $d$ \\
\hline MA $(8-40)$ & 28,91 & 6,12 & 32,02 & 5,29 & $-8,84$ & 0,00 & $-0,54$ \\
\hline MRS (6-30) & 20,82 & 5,20 & 20,59 & 5,47 & 0,703 & n.s. & - \\
\hline ML (6-30) & 24,47 & 4,30 & 26,36 & 3,36 & $-7,99$ & 0,00 & $-0,49$ \\
\hline Chicos & M & DT & M & DT & $t_{511}$ & $p$ & $d$ \\
\hline MA $(8-40)$ & 28,87 & 6,11 & 32,32 & 5,91 & $-5,32$ & 0,00 & $-0,59$ \\
\hline $\operatorname{MRS}(6-30)$ & 20,80 & 5,10 & 20,99 & 5,58 & $-0,34$ & n.s. & - \\
\hline ML (6-30) & 24,42 & 4,26 & 26,21 & 3,43 & $-4,09$ & 0,00 & $-0,44$ \\
\hline Chicas & M & DT & M & DT & $t_{543}$ & $p$ & $d$ \\
\hline MA $(8-40)$ & 29,04 & 6,18 & 31,94 & 5,12 & $-5,17$ & 0,00 & $-0,54$ \\
\hline MRS (6-30) & 20,86 & 5,56 & 20,48 & 5,44 & 0,673 & n.s. & - \\
\hline ML (6-30) & 24,63 & 4,44 & 26,40 & 3,35 & $-4,70$ & 0,00 & $-0,49$ \\
\hline 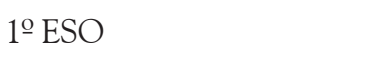 & M & DT & M & DT & $t_{304}$ & $p$ & $\mathrm{~d}$ \\
\hline MA (8-40) & 29,49 & 6,14 & 32,57 & 5,60 & $-4,45$ & 0,00 & $-0,51$ \\
\hline MRS (6-30) & 21,35 & 5,03 & 20,89 & 5,34 & 0,76 & n.s. & - \\
\hline ML (6-30) & 24,10 & 4,54 & 26,31 & 3,16 & $-4,672$ & 0,00 & $-0,53$ \\
\hline $2^{\circ} \mathrm{ESO}$ & M & DT & M & DT & $t_{271}$ & $p$ & $d$ \\
\hline MA $(8-40)$ & 28,56 & 6,11 & 31,33 & 5,52 & $-3,90$ & 0,00 & $-0,47$ \\
\hline MRS (6-30) & 21,10 & 5,12 & 20,60 & 5,28 & 0,79 & n.s. & - \\
\hline ML (6-30) & 24,65 & 4,17 & 26,23 & 3,61 & $-3,31$ & 0,00 & $-0,40$ \\
\hline $3^{\circ} \mathrm{ESO}$ & M & DT & M & DT & $t_{250}$ & $\mathrm{p}$ & d \\
\hline MA $(8-40)$ & 28,57 & 6,15 & 31,54 & 5,61 & $-3,99$ & 0,00 & $-0,54$ \\
\hline MRS (6-30) & 20,44 & 5,38 & 20,90 & 5,72 & $-0,66$ & n.s. & - \\
\hline ML (6-30) & 24,82 & 3,90 & 26,31 & 3,69 & $-3,11$ & 0,00 & $-0,51$ \\
\hline
\end{tabular}




\begin{tabular}{lccccccc}
\hline \multicolumn{1}{c}{ Metas académicas (rango) } & \multicolumn{2}{c}{ Baja conducta rosocial } & \multicolumn{2}{c}{ Alta conducta prosocial } & \multicolumn{3}{c}{ Significación estadística } \\
\hline \multicolumn{1}{c}{ Muestra total } & $\mathrm{M}$ & $\mathrm{DT}$ & $\mathrm{M}$ & $\mathrm{DT}$ & $t_{1056}$ & $p$ & $d$ \\
\hline $4^{\text {o }}$ ESO & $\mathrm{M}$ & $\mathrm{DT}$ & $\mathrm{M}$ & $\mathrm{DT}$ & $t_{225}$ & $p$ & $d$ \\
MA (8-40) & 28,69 & 6,04 & 32,56 & 4,44 & $-5,42$ & 0,00 & $-0,77$ \\
MRS (6-30) & 19,46 & 5,35 & 20,05 & 5,51 & $-0,76$ & n.s. & - \\
ML (6-30) & 24,47 & 4,54 & 26,54 & 3,01 & $-4,08$ & 0,00 & $-0,58$ \\
\hline
\end{tabular}

Nota. MA = Metas de Aprendizaje, MRS = Metas de Refuerzo Social, ML = Metas de Logro, ESO = Educación Secundaria Obligatoria, n.s. $=$ no significativo.

Fuente: elaboración propia.

mer caso y pequeña en el segundo. Sin embargo, no se hallan diferencias estadísticamente significativas entre ambos grupos en Metas de Refuerzo Social. Este patrón de resultados es similar en chicos y chicas. Del mismo modo, los resultados respecto a los cursos académicos indican que los estudiantes con alta conducta prosocial de todos los cursos de la ESO puntúan significativamente más alto que sus iguales con baja conducta prosocial en las escalas de Metas de Aprendizaje y Metas de Logro, siendo el tamaño del efecto moderado en todos los casos excepto en 2 o de ESO, donde las diferencias presentan una magnitud pequeña. Una vez más, no se encuentran diferencias estadísticamente significativas en Metas de Refuerzo Social entre los dos grupos comparados en cada uno de los cursos académicos.

\section{¿Es la conducta prosocial un predictor de las} metas académicas?

En la Tabla 2 se presentan los resultados derivados de la regresión logística binaria para la probabilidad de presentar altas Metas de Aprendizaje y Metas

TABLA 2

Regresión logística para la probabilidad de metas académicas

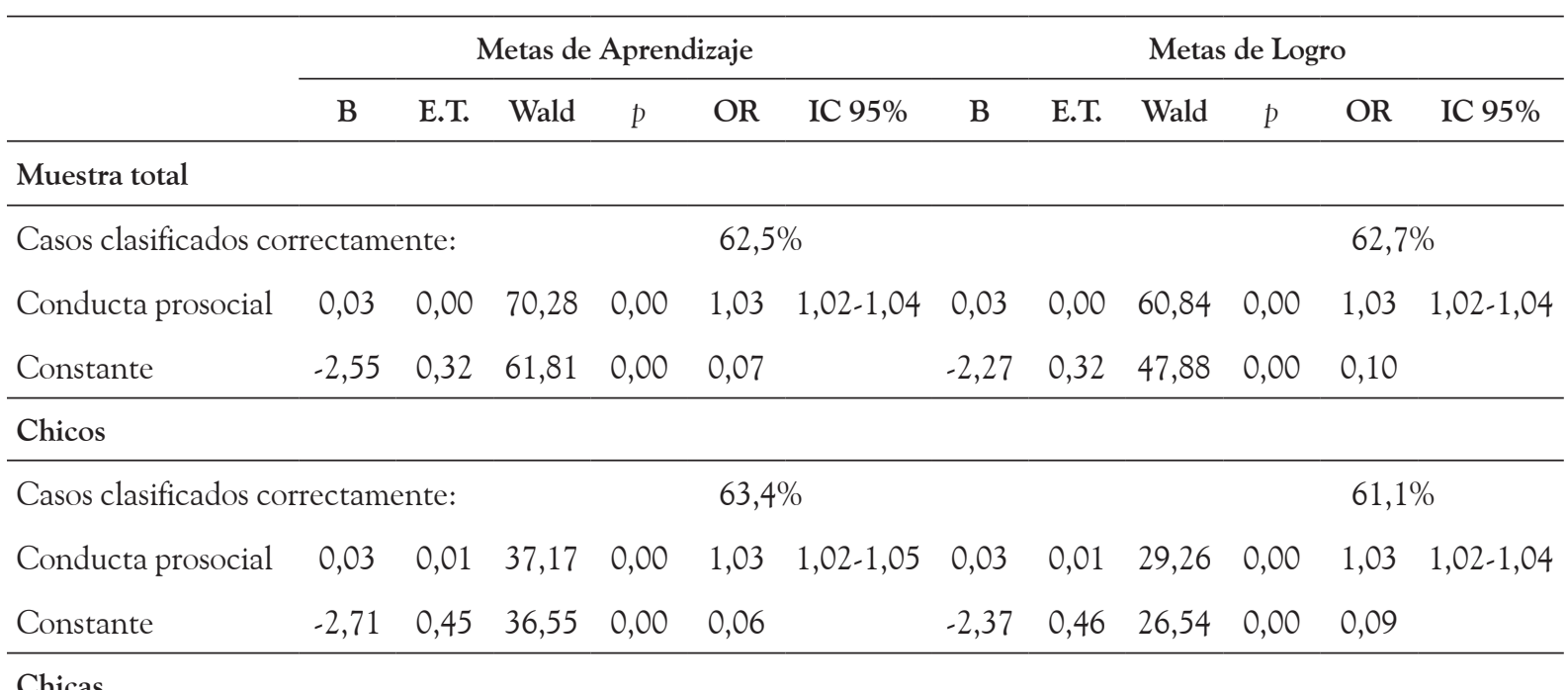

Chicas

Casos clasificados correctamente:

$62,9 \%$

$65,6 \%$ 


\begin{tabular}{|c|c|c|c|c|c|c|c|c|c|c|c|c|}
\hline & \multicolumn{6}{|c|}{ Metas de Aprendizaje } & \multicolumn{6}{|c|}{ Metas de Logro } \\
\hline & B & E.T. & Wald & $p$ & OR & IC $95 \%$ & B & E.T. & Wald & $p$ & OR & IC $95 \%$ \\
\hline Conducta prosocial & 0,03 & 0,01 & 27,74 & 0,00 & 1,04 & $1,02-1,05$ & 0,03 & 0,01 & 24,75 & 0,00 & 1,03 & $1,02-1,04$ \\
\hline Constante & $-2,60$ & 0,56 & 21,78 & 0,00 & 0,07 & & $-2,31$ & 0,56 & 17,06 & 0,00 & 0,09 & \\
\hline \multicolumn{13}{|l|}{$1^{\circ} \mathrm{ESO}$} \\
\hline \multicolumn{3}{|c|}{ Casos clasificados correctamente: } & \multicolumn{4}{|c|}{$61,6 \%$} & & & & \multicolumn{3}{|c|}{$64,7 \%$} \\
\hline Conducta prosocial & 0,03 & 0,01 & 23,65 & 0,00 & 1,03 & $1,02-1,05$ & 0,03 & 0,01 & 20,64 & 0,00 & 1,03 & $1,02-1,05$ \\
\hline Constante & $-2,52$ & 0,58 & 19,00 & 0,00 & 0,08 & & $-2,70$ & 0,59 & 21,07 & 0,00 & 0,06 & \\
\hline \multicolumn{13}{|l|}{ 2을 } \\
\hline \multicolumn{3}{|c|}{ Casos clasificados correctamente: } & \multicolumn{4}{|c|}{$63,4 \%$} & & & & \multicolumn{3}{|c|}{$62,6 \%$} \\
\hline Conducta prosocial & 0,03 & 0,01 & 13,49 & 0,00 & 1,03 & $1,02-1,04$ & 0,03 & 0,01 & 15,31 & 0,00 & 1,03 & $1,02-1,04$ \\
\hline Constante & $-2,20$ & 0,57 & 14,77 & 0,00 & 0,11 & & $-2,10$ & 0,61 & 11,68 & 0,00 & 0,12 & \\
\hline \multicolumn{13}{|l|}{ 3릉 } \\
\hline \multicolumn{3}{|c|}{ Casos clasificados correctamente: } & \multicolumn{4}{|c|}{$57,8 \%$} & & & & \multicolumn{3}{|c|}{$61,7 \%$} \\
\hline Conducta prosocial & 0,03 & 0,01 & 12,74 & 0,00 & 1,03 & $1,02-1,05$ & 0,03 & 0,01 & 10,45 & 0,00 & 1,03 & $1,02-1,04$ \\
\hline Constante & $-2,51$ & 0,71 & 12,55 & 0,00 & 0,08 & & $-1,91$ & 0,72 & 7,04 & 0,01 & 0,15 & \\
\hline \multicolumn{13}{|l|}{$4^{\circ} \mathrm{ESO}$} \\
\hline \multicolumn{3}{|c|}{ Casos clasificados correctamente: } & \multicolumn{4}{|c|}{$66,5 \%$} & & & & \multicolumn{3}{|c|}{$65,8 \%$} \\
\hline Conducta prosocial & 0,04 & 0,01 & 18,95 & 0,00 & 1,04 & $1,02-1,06$ & 0,03 & 0,01 & 8,07 & 0,00 & 1,03 & $1,02-1,04$ \\
\hline Constante & $-3,22$ & 0,85 & 14,24 & 0,00 & 0,04 & & $-1,69$ & 0,79 & 4,51 & 0,03 & 0,18 & \\
\hline
\end{tabular}

Nota. $\mathrm{MA}=$ Metas de Aprendizaje, MRS = Metas de Refuerzo Social, ML = Metas de Logro, ESO = Educación Secundaria Obligatoria B = coeficiente; E.T. = error estándar; $p$ = probabilidad; OR = odd ratio; I.C. = intervalo de confianza al 95\%.

Fuente: elaboración propia.

de Logro en la muestra total, así como en las muestras por género y curso académico a partir de la puntuación en conducta prosocial. Los datos han permitido crear modelos de regresión logística, para la muestra total, ambos géneros y todos los cursos académicos, permitiendo hacer estimaciones correctas respecto a la probabilidad de presentar altas Metas de Aprendizaje, las cuales varían entre el 57,8 \% (modelo de 30 de ESO) y el 66,5\% (modelo de 4을 $\mathrm{ESO}$ ) y altas Metas de Logro, las cuales oscilan entre el 61,1\% (modelo chicos) y el 65,8 \% (modelo de 4⿳⺈ de ESO). Sin embargo, los datos no han permitido crear modelos de regresión logística respecto a la probabilidad de presentar altas Metas de Refuerzo Social en la muestra total y en todas las submuestras analizadas por género y curso académico. Las odd ratio (OR) obtenidas revelaron que la probabilidad de presentar altas metas de aprendizaje y de logro es, según la muestra analizada, 1,03 y 1,04 veces mayor por cada punto que incrementa la puntuación en conducta prosocial, es decir, la probabilidad de presentar altas metas de aprendizaje y de logro aumenta un $3 \%$ en la muestra total y en el resto de submuestras analizadas, excepto en las chicas y los estudiantes de $4^{\circ}$ de ESO donde la probabilidad de presentar altas metas de aprendizaje aumenta un $4 \%$. 


\section{¿Predicen las metas académicas la conducta prosocial?}

En este caso, los datos permitieron crear modelos de regresión logística para la muestra total y para todas las submuestras por género y curso académico, permitiendo hacer estimaciones correctas respecto a la probabilidad de presentar alta conducta prosocial (rango: $62,7 \%-78,9 \%$ ) a partir de las puntuaciones en metas académicas (véase Tabla 3).

En cuanto a la muestra total, las $\mathrm{OR}$ revelaron que la probabilidad de presentar alta conducta prosocial es 1,10 y 1,12 veces mayor por cada punto que incrementa la puntuación en metas de aprendizaje y metas de logro, respectivamente, mientras que es, 91 veces menor por cada punto que incrementa la puntuación en metas de refuerzo social, es decir, la probabilidad de presentar alta conducta prosocial aumenta un $10 \%$ y un $12 \%$ cuando se tiende a adoptar metas de aprendizaje y metas de logro, respectivamente, mientras que disminuye un $9 \%$ cuando se persiguen metas de refuerzo social.

Para la muestra de chicos, las OR indicaron que la probabilidad de presentar alta conducta prosocial aumenta un $11 \%$ cuando se adoptan metas de aprendizaje y disminuye un $7 \%$ cuando se adoptan metas de refuerzo social. Además, en la muestra de chicas, las OR indicaron que la probabilidad de presentar alta conducta prosocial aumenta un $9 \%$ y un $11 \%$ cuando se adoptan metas de aprendizaje y de logro, respectivamente, mientras que disminuye un $9 \%$ cuando se persiguen metas de refuerzo social.

Para la muestra de estudiantes de $1^{\circ}$ de ESO, las OR indicaron que la probabilidad de presentar alta conducta prosocial aumenta un $8 \%$ cuando se adoptan metas de aprendizaje y un $16 \%$ cuando se adoptan metas de logro, mientras que disminuye un $11 \%$ cuando se adoptan metas de refuerzo social.

Respecto a los estudiantes de $2^{\circ}$ de ESO, las OR revelaron que la probabilidad de presentar alta conducta prosocial aumenta un $12 \%$ cuando se adoptan metas de aprendizaje y disminuye un $7 \%$ cuando se persiguen metas de refuerzo social.

En cuanto a la muestra de de 3 $\mathrm{ESO}$, las OR mostró que la probabilidad de presentar alta conducta prosocial aumenta un 9\% cuando los estudiantes orientan su estudio hacia metas de aprendizaje.

Por último, las OR correspondientes a la muestra de $4^{\circ}$ de ESO revelaron que la probabilidad de presentar alta conducta prosocial aumenta un $17 \%$ y un $12 \%$ cuando se adoptan metas de aprendizaje y de logro, respectivamente, aunque disminuye un $10 \%$ cuando se persiguen metas de refuerzo social.

TABLA 3

Regresión logística para la probabilidad de alta conducta prosocial

\begin{tabular}{|c|c|c|c|c|c|c|}
\hline & B & E.T. & Wald & $p$ & OR & IC $95 \%$ \\
\hline \multicolumn{7}{|c|}{ Muestra total } \\
\hline \multicolumn{7}{|c|}{ Casos clasificados correctamente: $65,6 \%$} \\
\hline MA & 0,09 & 0,01 & 39,52 & 0,00 & 1,10 & $1,06-1,33$ \\
\hline MRS & $-0,09$ & 0,01 & 38,95 & 0,00 & 0,91 & $0,88-0,93$ \\
\hline ML & 0,11 & 0,02 & 23,35 & 0,00 & 1,12 & $1,06-1,16$ \\
\hline Constante & $-3,67$ & 0,48 & 58,26 & 0,00 & 0,03 & \\
\hline \multicolumn{7}{|l|}{ Chicos } \\
\hline \multicolumn{7}{|c|}{ Casos clasificados correctamente: $78,4 \%$} \\
\hline MA & 0,13 & 0,02 & 31,44 & 0,00 & 1,14 & $1,08-1,19$ \\
\hline
\end{tabular}




\begin{tabular}{|c|c|c|c|c|c|c|}
\hline & B & E.T. & Wald & $p$ & OR & IC 95\% \\
\hline MRS & $-0,06$ & 0,02 & 6,88 & 0,01 & 0,93 & $0,89-0,98$ \\
\hline Constante & $-3,94$ & 0,68 & 34,01 & 0,00 & 0,02 & \\
\hline \multicolumn{7}{|l|}{ Chicas } \\
\hline \multicolumn{7}{|c|}{ Casos clasificados correctamente: $78,9 \%$} \\
\hline MA & 0,09 & 0,02 & 14,33 & 0,00 & 1,09 & $1,04-1,15$ \\
\hline MRS & $-0,10$ & 0,03 & 14,08 & 0,00 & 0,91 & $0,86-0,95$ \\
\hline ML & 0,10 & 0,04 & 8,05 & 0,00 & 1,11 & $1,03-1,18$ \\
\hline Constante & $-2,19$ & 0,74 & 8,83 & 0,00 & 0,11 & \\
\hline \multicolumn{7}{|l|}{$1^{\mathrm{o}} \mathrm{ESO}$} \\
\hline \multicolumn{7}{|c|}{ Casos clasificados correctamente: $66 \%$} \\
\hline MA & 0,09 & 0,03 & 7,29 & 0,01 & 1,08 & $1,02-1,14$ \\
\hline MRS & $-0,11$ & 0,03 & 13,85 & 0,00 & 0,89 & $0,84-0,94$ \\
\hline ML & 0,14 & 0,04 & 10,61 & 0,00 & 1,16 & $1,05-1,26$ \\
\hline Constante & $-4,14$ & 0,92 & 19,99 & 0,00 & 0,02 & \\
\hline \multicolumn{7}{|l|}{$2^{2} \mathrm{ESO}$} \\
\hline \multicolumn{7}{|c|}{ Casos clasificados correctamente: $63,7 \%$} \\
\hline MA & 0,11 & 0,03 & 19,63 & 0,00 & 1,12 & $1,06-1,17$ \\
\hline MRS & $-0,08$ & 0,03 & 7,45 & 0,01 & 0,93 & $0,87-0,97$ \\
\hline Constante & $-1,97$ & 0,72 & 7,55 & 0,01 & 0,14 & \\
\hline \multicolumn{7}{|l|}{ 3 $\mathrm{ESO}$} \\
\hline \multicolumn{7}{|c|}{ Casos clasificados correctamente: $62,7 \%$} \\
\hline MA & 0,09 & 0,02 & 14,22 & 0,00 & 1,09 & $1,04-1,13$ \\
\hline Constante & $-2,38$ & 0,70 & 11,62 & 0,00 & 0,09 & \\
\hline \multicolumn{7}{|l|}{$4^{\circ} \mathrm{ESO}$} \\
\hline \multicolumn{7}{|c|}{ Casos clasificados correctamente: $74 \%$} \\
\hline MA & 0,16 & 0,04 & 16,10 & 0,00 & 1,17 & $1,08-1,26$ \\
\hline MRS & $-0,10$ & 0,04 & 6,76 & 0,00 & 0,90 & $0,83-0,97$ \\
\hline ML & 0,11 & 0,05 & 4,58 & 0,03 & 1,12 & $1,01-1,24$ \\
\hline Constante & $-4,92$ & 1,21 & 16,63 & 0,00 & 0,01 & \\
\hline
\end{tabular}

Nota. MA = Metas de Aprendizaje, MRS = Metas de Refuerzo Social, ML = Metas de Logro, B = coeficiente; E.T. = error estándar; $g l=$ grados de libertad; $p=$ probabilidad; $O R=$ odd ratio; I.C. = intervalo de confianza al 95\%; ESO = Educación Secundaria Obligatoria.

Fuente: elaboración propia. 


\section{Discusión}

El presente estudio tuvo un doble objetivo. En primer lugar, analizar las diferencias en metas de aprendizaje, metas de logro y metas de refuerzo social entre estudiantes con baja y alta conducta prosocial. En segundo lugar, examinar la capacidad predictiva recíproca entre conducta prosocial y metas académicas en una muestra de estudiantes españoles de ESO.

Tal y como se estableció en la primera hipótesis, los resultados derivados de las pruebas de significación estadística revelaron que los estudiantes prosociales, comparados con sus iguales no prosociales, puntuaron significativamente más alto en metas de aprendizaje y logro. Además, este patrón de resultados fue hallado tanto en la muestra total como en ambos géneros y en todos los cursos de ESO. Del mismo modo, consistente con la segunda hipótesis, este estudio también reveló que la conducta prosocial fue un predictor positivo y estadísticamente significativo de altas metas de aprendizaje y logro. De este modo, la probabilidad de presentar altas metas de aprendizaje y de logro aumentó entre un $3 \%$ y un $4 \%$, dependiendo de la muestra analizada, por cada punto que incrementó la puntuación en conducta prosocial. Inversamente, la tercera hipótesis de este estudio estableció que las puntuaciones en metas de aprendizaje y logro serían predictores positivos y estadísticamente significativos de la probabilidad de presentar alta conducta prosocial. Los resultados permitieron confirmar esta hipótesis ya que esta probabilidad aumentó entre un 8 \% (1을 $\mathrm{ESO}$ ) y un 17 \% (4은 de ESO) cuando los estudiantes adoptaron metas de aprendizaje, mientras que aumentó un $12 \%$ cuando los estudiantes adoptaron metas de logro (muestra total). Sin embargo, las metas de logro no fueron un predictor estadísticamente significativo de la elevada conducta prosocial de las muestras en las muestras de chicos y de $3{ }^{\circ}$ y $4^{\circ}$ de ESO. En cualquier caso, estos resultados ponen de manifiesto que los estudiantes con alta prosociabilidad hacia sus iguales son también los que tienen una mayor motivación para aprender y para conseguir buenos resultados académicos, y viceversa. De hecho, si se tuviese que elaborar un perfil a partir de estas características sería el de un estudiante verdaderamente ideal: quiere y le interesa aprender, quiere conseguir buenos resultados académicos y, además, ayuda, coopera y empatiza con sus iguales y tiende a cumplir con las normas establecidas en el aula. De esta forma, los resultados del presente estudio parecen apoyar, una vez más, la teoría del contenido de las metas o las metas múltiples propuesta por Wentzel (2005). Según esta perspectiva teórica, las metas académicas y sociales de los estudiantes están relacionadas y la coordinación de éstas contribuye de manera significativa al éxito escolar. Sin embargo, la naturaleza transversal de este estudio impide el establecimiento de inferencias causales respecto a la forma en como se relacionan las metas sociales y académicas de los estudiantes. En este sentido, la teoría de Wentzel mantiene que las metas académicas (metas de aprendizaje y logro) y sociales (comportarse de manera prosocial y socialmente responsable) pueden relacionarse de tres formas distintas; (a) las metas académicas y las metas sociales son independientes. Así, ambos tipos de metas operan independientemente, aunque de forma complementaria; (b) las metas académicas tiene su origen en las metas sociales; o (c) las metas académicas y sociales mantienen relaciones jerárquicas y recíprocas de forma que un estudiante puede hacer las tareas escolares correctamente con el fin de cooperar con los iguales y profesores y cumplir con las reglas establecidas en el aula, o bien, un estudiante puede creer que comportándose de forma prosocial y socialmente responsable lo conducirá hacia un interés intrínseco por las tareas escolares y a orientarse hacia la búsqueda del éxito académico. Por tanto, futuras investigaciones deberían analizar la relación entre estas variables mediante el empleo de una metodología causal desde el modelado de ecuaciones estructurales.

En cuanto a la relación entre conducta prosocial y metas de refuerzo social, los resultados de este estudio no permitieron apoyar empíricamente la cuarta y quinta hipótesis, ya que no se hallaron diferencias estadísticamente significativas en metas de refuerzo social entre los estudiantes con baja 
y alta conducta prosocial. Además, la conducta prosocial no fue un predictor estadísticamente significativo de las metas de refuerzo social. Por tanto, la tendencia a la empatía, la ayuda, la cooperación y el altruismo hacia los demás que caracterizan el comportamiento prosocial (Eisenberg et al., 2006), no conlleva la búsqueda de un reconocimiento social por parte de los demás. Así, podría considerarse que la tendencia a manifestar una conducta prosocial no se activa con el fin de agradar y evitar el rechazo de los demás, sino que más bien esta tendencia es inherente la propia persona. Sin embargo, de acuerdo en la sexta hipótesis, las metas de refuerzo social fueron un predictor negativo y estadísticamente significativo respecto a la probabilidad de presentar una elevada puntuación en conducta prosocial, excepto en la muestra de $3^{\circ}$ de $\mathrm{ESO}$, de manera que esta probabilidad disminuye entre un $7 \%$ (chicos y 2 - de ESO) y un $12 \%$ (4을 ESO), cuando los estudiantes persiguen metas de refuerzo social. En este sentido, podría concluirse que aquellos estudiantes que buscan un refuerzo y un reconocimiento social son los que desarrollan los niveles más bajos de conducta prosocial hacia los iguales. En cierto modo, esto también puede interpretarse en el sentido de que aquellas personas con altas metas de refuerzo social están sobre todo preocupadas por su propia imagen personal ante los demás, mientras que las personas que muestran una alta conducta prosocial hacia los iguales están más preocupadas por ayudar a los demás sin pensar en su propia imagen, es decir, serían el prototipo de personas altruistas. En definitiva, sería la preocupación por uno mismo frente a la preocupación por los otros.

El presente estudio presenta algunas limitaciones que futuras investigaciones deberían examinar: (1) el uso del CEMA como medida de las metas académicas no tiene en cuenta la diferenciación entre tendencias de aproximación y de evitación como dimensiones independientes, tanto en el caso de las metas de aprendizaje como en el caso de las metas de rendimiento (Pintrich, 2003). Por ello, futuros estudios deberían analizar cómo se relaciona la conducta prosocial con estos dos tipos de metas de aprendizaje; (2) aunque el muestreo empleado en este estudio garantiza la representatividad de la muestra, esta se compone únicamente de estudiantes de ESO, por lo que los resultados obtenidos en el mismo no pueden generalizarse a estudiantes de otros niveles educativos como Educación Infantil, Educación Primaria, Bachillerato y Educación Superior. Futuros trabajos deberían confirmar si los resultados hallados en ESO difieren o se mantienen en otros niveles educativos con el fin de incrementar la validez externa de estos hallazgos; y (3) es importante resaltar que en esta investigación se han empleado únicamente medidas de autoinforme, lo cual puede introducir sesgos derivados de la deseabilidad social, aspecto este particularmente importante cuando se evalúa la conducta prosocial. En este sentido, la investigación futura debería emplear distintos procedimientos de evaluación tales como, por ejemplo, nominaciones sociométricas y escalas de valoración por los iguales y profesores.

A pesar de estas limitaciones y consideraciones, el presente estudio puede considerarse pionero ya que es la primera vez que se ha demostrado la existencia de una relación positiva y estadísticamente significativa entre conducta prosocial autoinformada y metas académicas en estudiantes españoles de ESO

Por último, los resultados del presente estudio sugieren algunas reflexiones tanto para los profesores, como para los profesionales e investigadores de la Educación y la Psicología. Por ejemplo, las sucesivas reformas educativas realizadas durante las últimas décadas se han centrado en la motivación académica y en las calificaciones escolares de los estudiantes de ESO, sin profundizar en el conocimiento relativo a la influencia que pueden tener diversos factores sociales o contextuales sobre las mismas. En este sentido, los resultados de este estudio han demostrado la existencia de interrelaciones entre el comportamiento prosocial y la motivación escolar, lo que confirma lo argumentado por otros autores quienes indican que la comunidad educativa debería atender a una variedad de factores interpersonales, incluyendo el establecimiento de relaciones sociales positivas o prosociales con los iguales, además de seguir enfatizando sobre diversas variables intrapersonales 
como la motivación escolar y el rendimiento académico (Pintrich, 2003).

\section{Referencias}

Alderman, M. K. (2008). Motivation for achievement: Possibilities for teaching and learning (3rd ed.). Nueva York, NY: Taylor \& Francis.

Bandura, A., Caprara, G. V., Barberanelli, C., Gerbino, M. \& Pastorelli, C. (2003). Role of affective selfregulatory efficacy in diverse sphere of psychosocial functioning. Child Development, 74, 769-782.

Covington, M. V. (2000). Goal theory, motivation, and school achievement: An integrative review. Annual Review of Psychology, 51, 171-200.

Delgado, B., Inglés, C. J., García-Fernández, J. M., Castejón, J. L. \& Valle, A. (2010). Diferencias de género y curso en metas académicas en alumnos de educación secundaria obligatoria. Revista Española de Pedagogía, 245, 67-84.

De Maris, A. (2003). Logistic regression. In J. A. Schinka \& W. F. Velicer (Eds.), Research Methods in Psychology (pp. 509-532). New Jersey, NJ: John Wiley \& Sons.

Eisenberg, N., Fabes, R. A. \& Spinrad, T. L. (2006). Prosocial development. In W. Damon \& N. Eisenberg (Eds.), Handbook of Child Psychology: Social, Emotional and Personality Development (Vol. 3, pp. 646-718). Nueva York: John Wiley \& Sons.

Furrer, C. \& Skinner, E. (2003). Sense of relatedness as a factor in children's academic engagement and performance. Journal of Educational Psychology, 95, 148-162.

Gilman, R. \& Anderman, E. M. (2006). The relationship between relative levels of motivation and intrapersonal, interpersonal, and academic functioning among older adolescents. Journal of School Psychology, 44, 375-391.

Hayamizu, T. \& Weiner, B. (1991). A test of Dwek's model of achievement goals are related to perceptions of ability. Journal of Experiemental Education, $59,226-234$.

Hedges, L. V. (1981). Distribution theory for Glass's estimator of effect size and related estimators. Journal of Educational Statistics, 6, 107-128.
Inderbitzen, H. M. \& Foster, S. L. (1992). The Teenage Inventory of Social Skills: Development, reliability, and validity. Psychological Assessment, 4, 451-459.

Inglés, C. J., Benavides, G., Redondo, J., García-Fernández, J. M., Ruiz-Esteban, C., Estévez, C. \& Huescar, E. (2009). Conducta prosocial y rendimiento académico en estudiantes españoles de Educación Secundaria Obligatoria. Anales de Psicología, 25, 93-101.

Inglés, C. J., García-Fernández, J. M., Castejón, J. L., Valle, A., Delgado, B. \& Marzo, J. C. (2009). Reliability and validity evidence of scores on the achievement goal tendencies questionnaire in a sample of Spanish students of compulsory secondary education. Psychology in the Schools, 46, 1048-1060.

Inglés, C. J., Hidalgo, M. D., Méndez, F. X. \& Inderbitzen, H. M. (2003). The Teenage Inventory of Social Skills: Reliability and validity of the Spanish translation. Journal of Adolescence, 26, 505-510.

Inglés, C. J., Martínez-Monteagudo, M. C., Delgado, B., Torregrosa, M. S., Redondo, J., Benavides, G. et al. (2008). Prevalencia de la conducta agresiva, conducta prosocial y ansiedad social en una muestra de adolescentes españoles: un estudio comparativo. Infancia y Aprendizaje, 31, 449-461.

Inglés, C. J., Delgado, B., García-Fernández, J. M., Ruiz-Esteban, C. \& Díaz-Herrero, A. (en prensa). Sociometric types and social interaction styles in a sample of Spanish adolescents. Spanish Journal of Psychology.

Ministerio de Educación y Ciencia (2007). Datos básicos de la Educación en España en el curso 2006/2007. Madrid: Autor.

Meece, J. L., Anderman, E. L. \& Anderman, L. H. (2006). Classroom goal structure, student motivation, and academic achievement. American Review of Psychology, 57, 487-503.

Pintrich, P. R. (2003). Motivation and classroom learning. In Reynolds \& Miller (Eds), Handbook of Psychology. Educational Psychology (Vol. 7, pp.103. 122). New Jersey, NJ: Wiley.

Rosa, A. I., Inglés, C. J., Olivares, J., Espada, J. P., Sánchez-Meca, J. \& Méndez, F. X. (2002). Eficacia 
del entrenamiento en habilidades sociales con adolescentes: de menos a más. Psicología Conductual, 10, 543-561.

Steinmayr, R. \& Spinath, B. (2009). The importance of motivation as a predictor of school achievement. Learning and Individual Differences, 19, 80-90.

Valle, A., Núñez, J. C., Rodríguez, S., Cabanach, R. G., González-Pienda, J. A. \& Rosario, P. (2010). Perfiles motivacionales y diferencias en variables afectivas, motivacionales y de logro. Universitas Psychologica, 9 (1), 109-121.

Wentzel, K. R. (2003). Sociometric status and adjustment in middle school: A longitudinal study. Journal of Early Adolescence, 23, 5-28.
Wentzel, K. R. (2005). Peer relationships, motivation, and academic performance at school. In J. Elliot \& C. S. Dweck (Eds.), Handbook of Competence and Motivation (pp. 279-296). Nueva York: Guilford Press.

Wentzel, K. R. \& Asher, S. R. (1995). The academic lives of neglected, rejected, popular, and controversial children. Child Development, 66, 754-763.

Wentzel, K. R. \& Watkins, D. E. (2002). Peer relationships and collaborative learning as contexts for academic enablers. School Psychology Review, 31, 366-377. 
